\title{
Cardiac disease in pregnancy
}

\author{
M. C. PETCh \\ M.A., M.D., M.R.C.P. \\ Department of Cardiology, Cambridge Area Health Authority (Teaching)
}

\begin{abstract}
Summary
The problems encountered in the management of pregnant patients with valvular, congenital and ischaemic heart disease are described with the help of illustrative cases. The increased incidence of cardiac dysrhythmias in pregnancy is also discussed.
\end{abstract}

Pregnancy results in an increase in blood volume and cardiac output and these changes are maintained until the time of delivery. The increase in cardiac output is mediated by an increase in both heart rate and stroke volume (Lees, 1979). The normal cardiovascular system can tolerate this extra burden with ease but may develop features which give rise to the impression of cardiac disease. These symptoms and signs must be recognized in order that they may be distinguished from those due to heart disease.

Both exertional dyspnoea and ankle swelling are common symptoms in a normal pregnancy. They should not give rise to concern but should be dealt with by explanation and reassurance. On examination there is often a large volume, and even collapsing arterial pulse; the venous pressure may be elevated. These signs of a hyperdynamic circulation are also reflected on auscultation when there may be an ejection systolic murmur. As in children, this murmur is best heard at the left sternal edge and probably arises from the outflow tract of the right ventricle which lies directly underneath the stethoscope. It is particularly easy to hear in slender individuals and in the absence of any other abnormal auscultatory findings it should be ignored. A physiological third heart sound may also be heard; atrial and diastolic flow murmurs have been reported, but these are rare.

For many years, interest in heart disease in pregnancy has centred on the problem of chronic rheumatic carditis (Szekely and Snaith, 1974). In most published series this has been the commonest form of heart disease encountered in the pregnant woman, and it is still an important cause of maternal mortality. It is true that rheumatic fever is now very uncommon in the U.K. and unlikely to be encountered during pregnancy. However, this does not apply to the immigrant population whose hearts seem to be particularly susceptible to the ravages of rheumatic fever. The risk of heart failure will increase as the pregnancy progresses and, at least in theory, is greatest at the time of delivery when the work of labour is superimposed on the increased cardiac output. Another dangerous time is immediately after delivery, because of a sudden further increase in blood volume. However, this danger is offset to some extent by the fact that the mother is under close medical supervision. Thus, the incidence of maternal heart failure is greater during the pregnancy when death may be sudden and unexpected. Patients with mitral stenosis are particularly vulnerable in this respect because of the increase in pulmonary blood volume associated with the increase in flow through the mitral valve and the tachycardia may suddenly result in pulmonary oedema, with few premonitory symptoms.

With the decline in rheumatic heart disease, it is now being recognized that many valve lesions which were previously attributed to rheumatic fever are in fact due to other pathologies, such as a congenital bicuspid aortic valve, or a floppy mitral valve. Although valve disease is becoming less common and mitral stenosis in particular may be disappearing, some cases will continue to be seen in antenatal clinics. The valve lesions will often take the form of mitral or aortic regurgitation and there are haemodynamic reasons which account for the relative safety of these conditions; for example in aortic regurgitation the tachycardia causes a reduction in regurgitant flow.

The management of patients with valve disease should ideally start before the pregnancy when decisions about surgery can be made. The majority of cases can be managed medically, but if valve replacement has to be undertaken, then it should probably be performed during the second trimester. Cardiopulmonary bypass is associated with an increased risk to the fetus but no increased risk to the mother. One consequence of the increasing safety of heart valve replacement has been an increase in the number of young people with prosthetic heart valves (Oakley and Doherty, 1976). Whenever possible, 
biological valves are to be preferred for women of child-bearing age, but if for any reason such a valve is not available then long-term anticoagulant therapy will be necessary. Warfarin is dangerous in early pregnancy because of teratogenesis and abortion; in late pregnancy there is the risk of fetal haemorrhage. Ideally, therefore, these patients should be anti-coagulated with long-term subcutaneous heparin which may be self-administered. If this is not possible, then warfarin may be continued and the risk accepted. However, most authorities advise substituting heparin at a late stage in the pregnancy.

Some of the difficulties in the management of patients with rheumatic heart disease before and during pregnancy are illustrated by the following case history. The patient presented with chorea at the age of 11 years and had a subsequent attack the following year. Three years later she was admitted to hospital when the diagnosis of aortic and mitral regurgitation was confirmed. The question of anticoagulant therapy was discussed, but at that time her left atrium was small and it was not until 5 years later, at the age of 20 , that warfarin was finally started. The next year she was planning to get married and the question of her future family was raised; for this reason she underwent cardiac catheterization. This showed that the haemodynamic lesion was severe and at rest her pulmonary capillary pressure was $16 \mathrm{mmHg}$. She was therefore advised against pregnancy but at the same time her symptoms were not severe enough to merit double valve replacement. She remained tolerably well but became increasingly determined to have a baby. Despite medical advice she conceived at the age of 25 years and presented for antenatal care at about 6 weeks. The decision to allow her to proceed with the pregnancy was self evident; it was also decided that by this stage it was pointless to stop the warfarin and it was recommended that she continue this drug until 37 weeks when heparin would be substituted. However, she aborted at 10 weeks.

Her resolve to have a baby was undiminished and this point of view could not be resisted. The warfarin was stopped and subcutaneous heparin was started in the hope that she would conceive shortly afterwards. She achieved this and all went well until about 10 weeks when she lapsed into atrial fibrillation with a fast ventricular response. This was partially controlled by treatment with digoxin and verapamil and at this stage the surgeons were consulted; it was agreed that her medical management should continue but that valve replacement should be undertaken if there was any deterioration. In the event, surgery was not necessary but her pregnancy was subsequently complicated by a severe attack of chicken pox. At 29 weeks her membranes ruptured spontaneously. Labour proceeded slowly and the following day a Caesarean section was performed; she was delivered of a live girl who weighed $1 \cdot 14 \mathrm{~kg}$. The child is developing normally.

This case also illustrates that the management of patients with heart disease during their pregnancy is best achieved by close co-operation between obstetrician and cardiologist and by careful supervision throughout, with admission into hospital and rest as necessary. Problems in management are most frequently encountered in those patients who have not had adequate medical supervision in the antenatal period. Even so, sterilization and abortion are rarely indicated, and it is seldom necessary to interfere with the obstetric management of the patient; for example, Caesarean section should only be performed for obstetric reasons. One other point in the medical management of these patients that merits attention is the use of ergometrine in the puerperium. Generally speaking, the risks of post-partum haemorrhage are greater than those of precipitating cardiac failure and therefore ergometrine should be used, albeit with caution.

Most of the simpler forms of congenital heart disease are now corrected before child-bearing age. This applies to such lesions as atrial and ventricular septal defect, patent ductus arteriosus, coarctation of aorta, Fallot's tetralogy and the more severe forms of aortic and pulmonary stenosis. Milder forms may be left alone but then these are not likely to cause trouble during pregnancy and indeed may only be recognized for the first time when the patient attends the antenatal clinic. More complex forms of congenital cyanotic heart disease have a low fertility and a high abortion rate. In the presence of pulmonary hypertension, either primary or due to the Eisenmenger reaction, there is a high maternal mortality and these $\mathbf{2}$ diseases probably form the only cardiac indication for therapeutic abortion.

One final problem relating to patients with valve or congenital heart disease is the risk of infective endocarditis. Endocarditis is most uncommon following normal delivery but it may follow complicated deliveries. The likely infecting organism in these cases will be Gram-negative bacilli or anaerobic streptococci. Thus if prophylaxis is to be given it must be directed towards these organisms and the combination of ampicillin and gentamicin can be recommended (de Swiet, 1976).

Cardiac dysrhythmias are not uncommon in young people and women who suffer from episodes of paroxysmal tachycardia often find that these increase in severity and duration during pregnancy. Extrasystoles are also more common, but both these and the attacks of paroxysmal tachycardia are generally benign and can be managed in the usual way with anti-arrhythmic drugs; if necessary, cardioversion may be carried out. Occasionally a more malignant 
arrhythmia may present during pregnancy as the following case history shows. The 27 -year-old patient developed syncope and palpitation when 26 weeks pregnant. She admitted to episodes of palpitation for some years, probably dating from the age of 16 years. She had not experienced syncopes until 1 or 2 weeks before she was seen. There were no abnormal physical findings in the cardiovascular system apart from multiple extrasystoles; the ECG and a period of 24-hr ECG monitoring showed that she was having frequent attacks of ventricular tachycardia. Treatment with disopyramide was started and this abolished the runs of ventricular tachycardia, although she continued to suffer multiple extrasystoles. However, these were not associated with any impairment of consciousness. The remainder of her pregnancy was normal and she was delivered of a normal child. Disopyramide was stopped, she had no further syncopes and her extrasystoles diminished in frequency during the weeks after delivery. The details of this case are to be published separately but it seems likely that the pregnancy exacerbated a longstanding rhythm disturbance with alarming consequences, although the mechanism by which this occurred remains obscure.

Another form of heart disease in pregnancy is congestive cardiomyopathy. This is more common in tropical countries and tends to affect the older and multiparous woman. The heart failure often increases in severity with each pregnancy and may recur in later life. It tends to improve following delivery and can be controlled with the usual measures. Its aetiology is obscure.
In the U.K. ischaemic heart disease is becoming more common in young people and Oliver amongst others has drawn attention to the incidence of heart attacks in young women (Oliver, 1974). This may be attributed in part to the habit of cigarette smoking and the more widespread use of the contraceptive pill. Coronary artery disease may be manifest as angina, which is uncommon in young women; as ventricular damage, which is seldom extensive enough to embarrass the myocardium during pregnancy; or as a fatal dysrhythmia. This last complication is one that is to be feared during pregnancy and delivery because it will occur suddenly and without warning. Thus, patients who are known to have coronary artery disease should have ECG monitoring during labour so that any increase in ventricular irritability can be recognized and dealt with.

\section{Acknowledgment}

My thanks are due to Hugh Fleming for his help in the preparation of this manuscript.

\section{References}

DE SWIET, M. (1976) Pregnancy and maternal heart disease. British Journal of Hospital Medicine, 15, 353.

LEES, M.M. (1979) Central circulatory responses in normotensive and hypertensive pregnancy. Postgraduate Medical Journal, 55, 311.

OAKLeY, C. \& Doherty, P. (1976) Pregnancy in patients after valve replacement. British Heart Journal, 38, 1133.

OlIVER, M.F. (1974) Ischaemic heart disease in young women. British Medical Journal, 4, 253.

Szekely, P. \& SNATT, L. (1974) Heart Disease and Pregnancy. p. 53. Churchill Livingstone, Edinburgh \& London. 